introducing injury prevention measures. Males are more frequently exposed than females, heading rates increase with age, and there is substantial variation between players. Heading is a rare event in the youngest age groups, especially among females.

\section{HEAD IMPACT DOSES AND 'NO-GO' DEFICITS IN OLYMPIC AND NON-OLYMPIC SPORT ATHLETES}

${ }^{1}$ Adam Bartsch, ${ }^{2}$ Lori Glover, ${ }^{3}$ Jay Alberts, ${ }^{3}$ Jason Cruickshank, ${ }^{3}$ Elizabeth Jansen, ${ }^{3}$ Edward Benzel, ${ }^{3}$ Sergey Samorezov, ${ }^{4}$ Vincent Miele, ${ }^{5}$ Julian Bailes, ${ }^{6}$ Gerald McGinty, ${ }^{9}$ Steven Rowson, ${ }^{6}$ Christopher D'Lauro, ${ }^{8}$ Tyler Rooks, ${ }^{7}$ Kenneth Cameron, ${ }^{7}$ Megan Houston, ${ }^{9}$ Emily Kieffer, ${ }^{10}$ Laurel $\mathrm{Ng},{ }^{10}$ Kiran Mathews, ${ }^{14}$ Mikael Swaren, ${ }^{11}$ Norman Link, ${ }^{15}$ Missy Fraser, ${ }^{12}$ Jason P Mihalik, ${ }^{12}$ Johna Mihalik-Register, ${ }^{13} \mathrm{JT}$ Eckner, ${ }^{13}$ Annalise Lane. ${ }^{1}$ Prevent Biometrics, Minneapolis, USA; ${ }^{2}$ Fairview Sports Medicine, Minneapolis, USA; ${ }^{3}$ Cleveland Clinic, Cleveland, USA; ${ }^{4}$ University of Pittsburgh Medical Center, Pittsburgh, USA; ${ }^{5}$ NorthShore University Health System, Chicago, USA; ${ }^{6}$ USA Air Force Academy, Colorado Springs, USA; ${ }^{7}$ USA Military Academy, West Point, USA; ${ }^{8}$ USA Army Aeromedical Research Laboratory, Fort Rucker, USA; ${ }^{9}$ Virginia Polytechnic University, Blacksburg, USA; ${ }^{10}$ L3 Harris, San Diego, USA; ${ }^{11}$ University of California-Berkeley, Berkeley, USA; ${ }^{12}$ University of North Carolina, Chapel Hill, USA; ${ }^{13}$ University of Michigan, Ann Arbor, USA; ${ }^{14}$ Swedish Olympic Academy, Stockholm, Sweden; ${ }^{15}$ Texas State University, Frisco, USA

\subsection{6/bjsports-2021-IOC.13}

Background The relationship between head impact dose and observable functional deficits remains unclear. While studies have almost exclusively examined American football athletes, in Olympic athletes there are almost no data that explore this relationship.

Objective We aimed to use an impact monitoring mouthguard (IMM) to quantify head impact doses in Olympic and nonOlympic Sports, identifying high-energy impacts on video as 'No-go' per the NFL protocol.

Design Retrospective meta-analysis from American football, basketball, boxing, ice hockey, karate, lacrosse, mixed martial arts, rugby, tae-kwon-do, soccer.

Setting Sporting field

Patients (or Participants) 4500 impacts over 800 player-games.

Interventions (or Assessment of Risk Factors) Impact doses where the athlete was observed as 'no-go'.

Main Outcome Measurements Kinetic energy transfer (KE), risk-weighted exposure (RWE), peak scalar linear acceleration (PLA), peak scalar linear velocity (PLV), peak scalar angular acceleration (PAA), peak scalar angular velocity (PAV), impact location, impact direction, 'No-go' status.

Results The median KE, RWE, PLA, PAA, PLV and PAV was 5 $\mathrm{J}, 0.0002,20 \mathrm{~g}, 1500 \mathrm{rad} / \mathrm{s}^{2}, 10 \mathrm{rad} / \mathrm{s}$ and $1.5 \mathrm{~m} / \mathrm{s}$, respectively. American football athletes sustained the highest energy impact doses, boxers and mixed-martial artists sustained the highest cumulative dose for a day of competition. Ice hockey had the highest rate of 'no-go' impacts versus total impacts collected. Karate had the highest rotational kinematics. Of the nine (9) highest energy impacts to the side and rear of the head, all were 'no-go' impacts. Of the top eight (8) highest energy impacts to the front of the head, none were 'no-go' impacts.

Conclusions 'No-go' observations occurred in high energy impact doses to the rear and the sides of the head, while similar impact doses to the forehead seemed tolerable. Prospective Olympic athlete impact monitoring could help identify risky exposures.

\section{BIOMARKERS IN SERUM AFTER HEAD IMPACT EXPOSURE IN FOOTBALL}

${ }^{1,2}$ Stian Bahr Sandmo, ${ }^{3}$ Peter Filipcik, ${ }^{3}$ Martin Cente, ${ }^{3}$ Jozef Hanes, ${ }^{1}$ Thor Einar Andersen,

1,4,5Truls M Straume-Næsheim, ${ }^{1}$ Roald Bahr. 'Oslo Sports Trauma Research Center, Norwegian School of Sport Sciences, Oslo, Norway; ${ }^{2}$ Faculty of Medicine, University of Oslo, Oslo, Norway; ${ }^{3}$ Institute of Neuroimmunology, Slovak Academy of Sciences, Bratislava, Norway; ${ }^{4}$ Department of Orthopedic Surgery, Akershus University Hospital, Lørenskog, Norway; ${ }^{5}$ Department of Orthopedic Surgery, Haugesund Rheumatism Hospital, Haugesund, Norway

\subsection{6/bjsports-2021-IOC. 14}

Background The link between head-impact exposure in football and neurological sequelae remains controversial. Bloodbased biomarkers can provide valuable information in traumatic brain injuries, reflecting e.g. axonal damage.

Objective To investigate if repetitive headers or accidental head impacts in football could cause structural damage to the brain, detected as an increase in serum concentrations of neurofilament light $(\mathrm{NfL})$ or tau proteins.

Design Prospective cohort study.

Setting Elite division football.

Participants Male football players in the Norwegian premier league, including a total of 621 player seasons.

Independent Variables Short- and long-term exposures in football, with and without head impacts.

Main Outcome Measurements Baseline NfL and tau were measured in Norwegian professional football players in preseason. Then, the effect of short-term exposures (independent variable) was assessed by measuring biomarker levels (dependent variable) after three conditions: (1) high-intensity exercise, (2) repetitive headers, and (3) head-impact incidents in a match. The effect of long-term head impact exposure was assessed by comparing two groups with relative differences in previous concussions and headers at baseline (low vs. high levels).

Results We analyzed 354 samples. Mean $( \pm$ SD) NfL concentration was $6.8 \pm 2.6 \mathrm{pg} / \mathrm{mL}$; mean tau concentration was $1.2 \pm 0.7 \mathrm{pg} / \mathrm{mL}$. We observed no short-term effects on NfL after exposure from either of the three conditions. Tau levels rose in response to high-intensity exercise and repetitive headers, but not after accidental head-impact incidents; the highest absolute values were seen $1 \mathrm{~h}$ after high-intensity exercise. We did not detect any long-term effects on serum $\mathrm{NfL}$ or tau concentrations from previous concussions and headers.

Conclusions NfL and tau in serum were unaffected by head impacts in football, after both short-term and long-term exposure. Importantly, tau levels seem to rise in response to exercise, emphasizing the need for appropriate control groups in future studies. Our findings highlight important characteristics and limitations for using $\mathrm{NfL}$ and tau as biomarkers in sports.

\section{TACKLE CHARACTERISTICS ASSOCIATED WITH CONCUSSION IN BRITISH UNIVERSITY LEVEL RUGBY UNION}

${ }^{1}$ Simon Roberts, ${ }^{2}$ Simon Kemp, 'Luke Morgan, ${ }^{1,2}$ Keith Stokes. ${ }^{1}$ University of Bath, Bath, UK: ${ }^{2}$ Rugby Football Union, London, UK

10.1136/bjsports-2021-IOC.15 
Background Understanding the risk of concussion and how this injury occurs in rugby union match play can inform future injury reduction strategies. This has been investigated in professional rugby but not in the UK elite level pathway.

Objective Describe the incidence of concussion and characteristics of tackles resulting in concussion in British University rugby union.

Design A one-season prospective cohort study; team medical staff reported match play concussions. Matches were filmed and tackle-related concussions were analysed alongside 796 non-injurious 'control' tackles.

Setting British University and Colleges (BUCS) 'Super' rugby union: season 2018/19.

Participants Eight teams (341 players).

Independent variables Match exposure.

Main Outcome Measurements Injury incidence and characteristics of tackles associated with concussion.

Results There were 43 concussions in 154 team games; incidence was 14.0 per 1000 player match hours (95\% CI: 10.1-18.8) and mean absence was 23 days (95\% CI: 16.129.9). Thirty-four (79\%) concussions occurred in the tackle; 22 by the tackler and 12 by the ball carrier. Twenty-five tackles resulting in concussion could be conclusively identified on video (tackler: 16; ball carrier: 9). For control tackles, $5 \%$ resulted in initial impact to the head/neck and $18 \%$ to the shoulders of the ball carrier compared with tackles resulting in concussions to the ball carrier [head/ neck, 3 (33\%); shoulder, 2 (22\%)] or tackler [head/neck, 2 (13\%); shoulder, 3 (19\%)]. For tackler concussions, 3 (19\%) involved head-to-head contact (2\% of control tackles) and $4(25 \%)$ involved head-to-shoulder contacts $(6 \%$ of control). The ball carrier was travelling at high speed for $67 \%$ of ball carrier concussions, compared with $29 \%$ for control tackles.

Conclusions Concussion incidence in high-level university rugby union is slightly lower than professional rugby with most concussions occurring in the tackle. Lowering tackle height may help reduce concussion for the ball carrier and the tackler should prioritise head positioning away from the ball carrier's head and shoulders.

\section{\begin{tabular}{|l|l}
\hline 017 & DOES THE OSLO SPORTS TRAUMA RESEARCH CENTER
\end{tabular} SHOULDER INJURY PREVENTION PROGRAM AFFECT THE RISK FACTORS EXTERNAL ROTATION STRENGTH AND INTERNAL ROTATION RANGE OF MOTION? A RANDOMIZED CONTROLLED STUDY AMONG ADOLESCENT HANDBALL PLAYERS}

${ }^{1}$ Hilde Fredriksen, ${ }^{2}$ Ann Cools, ${ }^{1}$ Roald Bahr, 'Grethe Myklebust. 'Oslo Sports Trauma Research Center, Norwegian School of Sport Sciences, Oslo, Norway; ${ }^{2}$ Ghent University, Rehabilitation Sciences and Physiotherapy, Ghent, Belgium

\subsection{6/bjsports-2021-IOC.16}

Background Shoulder problems are common in handball, but preventable. However, player compliance remains a challenge, as the existing prevention program is time consuming.

Objective To assess the effect of the Oslo Sports Trauma Research Center shoulder injury prevention program on external rotation (ER) strength and internal rotation (IR) range of motion (ROM), believed to represent key risk factors for injury.
Design Randomised controlled trial, single blinded

Setting Youth handball players (16-18 yrs)

Participants Four youth handball teams (three female, one male, 57 players, mean age 17.1 yrs) were randomly selected from eligible teams in the Oslo region, and randomized to an intervention group (28 players) or control group (29 players). Interventions The Oslo Sports Trauma Research Center shoulder injury prevention program was implemented during regular handball warm-up three times a week for 18 weeks in the intervention group.

Main Outcome Measurements The main outcome variable was the between-group difference in ER strength and IR ROM change from baseline to post intervention. Isometric ER strength was measured with a handheld dynamometer and IR ROM with a digital goniometer.

Results Mean dominant shoulder isometric ER strength increased significantly both in the intervention (10\%) and the control group $(6 \%)$ during the intervention, but there was no significant group by time interaction (group difference: 0.06 $\mathrm{N} / \mathrm{kg}$ (95\% CI: -0.04 to 0.17 ). IR ROM did not change in either group during the intervention.

Conclusions The Oslo Sports Trauma Research Center shoulder injury prevention program did not affect the risk factors ER strength and IR ROM. The preventive effect of the program must therefore be due to other factors.

\section{SPRAINO ${ }^{\circledR}$ REDUCES THE RISK OF LATERAL ANKLE SPRAIN INJURY AMONG INDOOR SPORT ATHLETES: A PILOT RANDOMIZED CONTROLLED TRIAL WITH 510 PARTICIPANTS}

\begin{abstract}
1,2Filip Gertz Lysdal, ${ }^{3}$ Thomas Bandholm, ${ }^{4}$ Janne Tolstrup, ${ }^{5,6}$ Mikkel Clausen, ${ }^{3}$ Stephanie Mann, ${ }^{7}$ Pelle Petersen, ${ }^{2}$ Thor Grønlykke, ${ }^{1,8}$ Uwe Kersting, ${ }^{9,10}$ Eamonn Delahunt, ${ }^{3,6}$ Kristian Thorborg. 'Sport Sciences, Department of Health Science and Technology, Aalborg University, Aalborg, Denmark; ${ }^{2}$ Spraino ApS, Copenhagen, Denmark; ${ }^{3}$ Physical Medicine and Rehabilitation Research - Copenhagen (PMR-C), Department of Physical and Occupational Therapy, Clinical Research Centre, and Department of Orthopedic Surgery, Amager-Hvidovre Hospital, Copenhagen University, Hvidovre, Denmark; ${ }^{4}$ National Institute of Public Health, University of Southern Denmark, Copenhagen, Denmark; ${ }^{5}$ School of Physiotherapy, Faculty of Health and Technology, University College Copenhagen, Copenhagen, Denmark; ${ }^{6}$ Department of Orthopedic Surgery, Sports Orthopedic Research Center-Copenhagen (SORC-C), Amager-Hvidovre Hospital, Copenhagen University, Hvidovre, Denmark; ${ }^{7}$ Section for Surgical Pathophysiology 7621, Rigshospitalet, University of Copenhagen, Copenhagen, Denmark; ${ }^{8}$ Institute of Biomechanics and Orthopaedics, German Sport University Cologne, Cologne, Germany; ${ }^{9}$ School of Public Health, Physiotherapy and Sports Science, University College Dublin, Dublin, Ireland; ${ }^{10}$ Institute for Sport and Health, University College Dublin, Dublin, Ireland
\end{abstract}

\subsection{6/bjsports-2021-IOC.17}

Background High shoe-surface friction is a proposed risk factor for 'non-contact' lateral ankle sprain (LAS) injuries. Spraino ${ }^{\circledR}$ is a novel product that minimizes friction at the lateral edge of the shoe, thereby potentially mitigating the risk.

Objective To determine preliminary effect and safety of Spraino ${ }^{\circledR}$ when used to prevent LAS injury among indoor sport athletes.

Design A double-blinded, prospective, two-arm pilot randomised controlled trial (RCT). Participants were allocated (1:1) to Spraino ${ }^{\circledR}$ or to a 'do-as-usual' control group. The random allocation was concealed for investigators and participants. Group allocations were outcome-assessor-blinded.

Setting Indoor sports clubs competing at divisional- or league level in handball, badminton and basketball in Denmark. 